\title{
Classifications and existence of nonoscillatory solutions of second order nonlinear neutral differential equations
}

\author{
by WANTONG Li (Zhangye)
}

\begin{abstract}
A class of neutral nonlinear differential equations is studied. Various classifications of their eventually positive solutions are given. Necessary and/or sufficient conditions are then derived for the existence of these eventually positive solutions. The derivations are based on two fixed point theorems as well as the method of successive approximations.
\end{abstract}

1. Introduction. Neutral nonlinear differential equations of the form

$$
\left(r(t)(x(t)-p(t) x(t-\tau))^{\prime}\right)^{\prime}+f(t, x(t-\delta))=0
$$

have been studied by a number of authors [2]-[5]. In particular, $\mathrm{Lu}$ in [4] studied the equation

$$
(x(t)-p(t) x(t-\tau))^{\prime \prime}+f(t, x(t-\delta))=0
$$

and obtained classifications of its nonoscillatory solutions as well as necessary and/or sufficient conditions for their existence.

Here, we will assume that $\tau$ is a positive number, $\delta$ is a nonnegative number, $p(t) \in C\left(\left[t_{0}, \infty\right), \mathbb{R}_{+}\right)$and $0 \leq p(t) \leq p<1$ for $t \geq t_{0}$ and $r(t) \in$ $C\left(\left[t_{0}, \infty\right),(0, \infty)\right)$. We further assume that for any $t \geq t_{0}, f$ is continuous as a function of $x \in \mathbb{R}$ and $x f(t, x)>0$ for $x \neq 0, t \geq t_{0}$.

In this paper we consider the classification of all nonoscillatory solutions of (1) in the cases

$$
\int_{t_{0}}^{\infty} \frac{1}{r(u)} d u<\infty \text { and } \int_{t_{0}}^{\infty} \frac{1}{r(u)} d u=\infty
$$

and give necessary and/or sufficient conditions for their existence.

1991 Mathematics Subject Classification: Primary 34C15.

Key words and phrases: oscillation, neutral nonlinear differential equation, asymptotic behavior.

Supported by the Science Foundation of Gansu Province of China for the Young. 
Hereafter, the term solution of (1) is always used to denote a real function $x(t)$ satisfying (1) for which $\sup _{t \geq t_{1}}|x(t)|>0$ for any $t_{1} \geq t_{0}$. We assume that (1) always has such solutions. A solution of (1) is called nonoscillatory if it is eventually positive or eventually negative. Otherwise it is called oscillatory. Equation (1) is called oscillatory if all its solutions are oscillatory. We use the functions $R(s, t)$ and $R(s)$ defined by

$$
R(s, t)=\int_{s}^{t} \frac{1}{r(u)} d u \text { and } R(s)=\int_{s}^{\infty} \frac{1}{r(u)} d u,
$$

where $s \geq t_{0}$. We also let $R_{0}=\lim _{t \rightarrow \infty} R\left(t_{0}, t\right)$.

We shall say that condition $(\mathrm{H})$ is met if the following hold:

$\left(\mathrm{H}_{1}\right) x f(t, x)>0$ for $x \neq 0, t \geq t_{0}$, and $f\left(t, x_{1}\right) \geq f\left(t, x_{2}\right)$ for $x_{1} \geq x_{2}$ $>0$ or $x_{1} \leq x_{2}<0, t \geq t_{0}$;

$\left(\mathrm{H}_{2}\right) 0 \leq p(t)<p<1$ for $t \geq t_{0}$.

Let $x(t)$ be a solution of (1). We will define a function $y(t)$ associated with $x(t)$ by

$$
y(t)=x(t)-p(t) x(t-\tau), \quad t \geq t_{0} .
$$

Note that if $x(t)$ is eventually positive, then the fact that

$$
\left(r(t) y^{\prime}(t)\right)^{\prime}=-f(n, x(t-\tau))<0
$$

for all large $t$ implies that $y^{\prime}(t)$ is of constant positive or constant negative sign eventually. This fact, in turn, implies that $y(t)$ is eventually positive or eventually negative.

In the following sections, we first give some lemmas which are interesting in their own right and then discuss the existence and asymptotic behavior of nonoscillatory solutions of the differential equation (1) for $R_{0}<\infty$ and $R_{0}=\infty$ respectively.

2. Preparatory lemmas. In this section, we will give some lemmas which are important in proving our results in Sections 3 and 4.

Lemma 1. Suppose that $(\mathrm{H})$ holds. Let $x(t)$ be an eventually positive (negative) solution of (1) and let $y(t)$ be defined by $(2)$. Then $y^{\prime}(t)$ is eventually of constant sign.

Lemma 2. Suppose that $(\mathrm{H})$ holds. Let $x(t)$ be an eventually positive (negative) solution of (1) and let $y(t)$ be defined by (2).

(i) If $R_{0}=\infty$ and $\lim _{t \rightarrow \infty} x(t)=0$, then $y(t)$ is eventually increasing (decreasing) and negative (positive) and $\lim _{t \rightarrow \infty} y(t)=0$. 
(ii) If $\lim \sup _{t \rightarrow \infty} x(t)>0$ (resp. $\left.\lim \sup _{t \rightarrow \infty}(-x(t))>0\right)$, then $y(t)$ is eventually positive (respectively negative).

Proof. (i) Let $x(t)$ be an eventually positive solution of (1). If $\lim _{t \rightarrow \infty} x(t)=0$, then $\lim _{t \rightarrow \infty} y(t)=0$. Suppose to the contrary that $y(t)>0$ for all large $t$. Then $y^{\prime}(t)<0$ for all large $t$. From $\left(r(t) y^{\prime}(t)\right)^{\prime}<0$, we obtain

$$
y(t) \leq y(s)+r(s) y^{\prime}(s) \int_{s}^{t} \frac{1}{r(u)} d u=y(s)+r(s) y^{\prime}(s) R(s, t)
$$

for $t \geq s$, where $s \geq t_{0}$ such that $x(t)>0$ for $t \geq s$. Since $r(t) y^{\prime}(t)$ is eventually decreasing, we can choose $t_{1}$ so large that $r\left(t_{1}\right) y^{\prime}\left(t_{1}\right)<0$, thus, $r(s) y^{\prime}(s)<r\left(t_{1}\right) y^{\prime}\left(t_{1}\right)=-a_{1}$ for $s \geq t_{1}$. Therefore

$$
y(t) \leq y(s)-a_{1} R(s, t), \quad a_{1}>0 .
$$

If $R_{0}=\infty$, taking the limit as $t \rightarrow \infty$ on both sides of the last inequality and in view of $\lim _{t \rightarrow \infty} y(t)=0$, we see that

$$
y(s) \geq \infty,
$$

which is a contradiction.

(ii) Let $x(t)$ be an eventually positive solution of (1) and $\lim _{\sup } \operatorname{pu}_{t \rightarrow \infty} x(t)$ $>0$. Then $y(t)>0$. If not, we have $y(t)<0$ for all large $t$. If $x(t)$ is unbounded, then there exists a sequence $\left\{t_{k}\right\}$ which tends to infinity and is such that

$$
x\left(t_{k}\right)=\max _{t \leq t_{k}} x(t)
$$

and $\lim _{k \rightarrow \infty} x\left(t_{k}\right)=\infty$. Then, from (2), we have

$$
y\left(t_{k}\right)=x\left(t_{k}\right)-p\left(t_{k}\right) x\left(t_{k}-\tau\right) \geq x\left(t_{k}\right)(1-p) .
$$

From the above inequality we obtain $\lim _{k \rightarrow \infty} y\left(t_{k}\right)=\infty$. This is a contradiction. If $x(t)$ is bounded, then there is a sequence $\left\{t_{k}\right\}$ which tends to infinity and is such that

$$
\lim _{k \rightarrow \infty} x\left(t_{k}\right)=\limsup _{t \rightarrow \infty} x(t)=L>0 .
$$

Since $\lim _{k \rightarrow \infty} x\left(t_{k}-\tau\right) \leq L$, we have

$$
0 \geq \lim _{k \rightarrow \infty} y\left(t_{k}\right) \geq L(1-p)>0 .
$$

This is also a contradiction and the proof is complete.

Lemma 3. Suppose that $(\mathrm{H})$ holds. Let $x(t)$ be an eventually positive (negative) solution of (1) and let $y(t)$ be defined by (2). If $R_{0}=\infty$, then $y^{\prime}(t)>0$ (respectively $\left.y^{\prime}(t)<0\right)$ for all large $t$.

P r o o f. Suppose $x(t)$ is an eventually positive solution of (1) and let $y(t)$ be defined by (2). We assert that $y^{\prime}(t)>0$ for all large $t$. If $\lim _{t \rightarrow \infty} x(t)=0$, 
in view of part (i) of Lemma 2, the conclusion holds. If $\lim \sup _{t \rightarrow \infty} x(t)$ $>0$, by part (ii) of Lemma 2, we have $y(t)>0$ for all large $t$. Suppose to the contrary that $y^{\prime}(t)$ is eventually negative. Note that in view of (1), $\left(r(t) y^{\prime}(t)\right)^{\prime}=-f(t, x(t-\delta))<0$ for all large $t$. Thus there is $t_{1} \geq t_{0}$ such that $y(t)>0, y^{\prime}(t)<0$ and $\left(r(t) y^{\prime}(t)\right)^{\prime}<0$ for all $t \geq t_{1}$. Then

$$
y(t) \leq y\left(t_{1}\right)+r\left(t_{1}\right) y^{\prime}\left(t_{1}\right) \int_{t_{1}}^{t} \frac{1}{r(s)} d s \rightarrow-\infty,
$$

which is a contradiction. The proof is complete.

The following lemma is independent of equation (1), and is obtained by $\mathrm{Lu}$ in [4].

Lemma 4. Suppose that $0 \leq p(t) \leq p<1, x(t)>0$ (resp. $x(t)<0)$ and $y(t)$ is defined by $(2)$.

(i) If $\lim _{t \rightarrow \infty} p(t)=p_{0}\left(\right.$ with $\left.0 \leq p_{0}<1\right)$ and $\lim _{t \rightarrow \infty} y(t)=a$ (with $|a|<\infty)$, then $\lim _{t \rightarrow \infty} x(t)=a /\left(1-p_{0}\right)$.

(ii) If $\lim _{t \rightarrow \infty} y(t)=\infty$ (resp. $\left.-\infty\right)$, then $\lim _{t \rightarrow \infty} x(t)=\infty$ (resp. $\left.-\infty\right)$.

3. The case $R_{0}<\infty$. We have already remarked that if $x(t)$ is an eventually positive solution of (1), then $y(t)$ and $y^{\prime}(t)$ are also of constant sign eventually. These sign regularities provide additional asymptotic information as will be seen in the following two lemmas.

LEMma 5. Suppose that $(\mathrm{H})$ holds. If $x(t)$ is a nonoscillatory solution of (1), then $y(t)$ defined by (2) is eventually increasing or decreasing and $\lim _{t \rightarrow \infty} y(t)=L$ exists, where $L$ is a finite constant.

Proof. Suppose $x(t)$ is an eventually positive solution of (1). If $\lim _{t \rightarrow \infty} x(t)=0$, then $\lim _{t \rightarrow \infty} y(t)=0$. If $\limsup _{t \rightarrow \infty} x(t)>0$, by part (ii) of Lemma 2, we have $y(t)>0$ for all large $t$. Thus, there exists $t_{1} \geq t_{0}$ such that $x(t-\delta)>0$ and $y(t)>0$ for $t \geq t_{1}$. It follows from (1) that $\left(r(t) y^{\prime}(t)\right)^{\prime}<0$, that is,

$$
y(t)<y(s)+r(s) y^{\prime}(s) \int_{s}^{t} \frac{d u}{r(u)}
$$

for $t \geq s, s \geq t_{1}$. If there exists a $t_{2} \geq t_{1}$ such that $y^{\prime}\left(t_{2}\right) \leq 0$, then it follows from (5) that $y(t)<y(s)$ for $t>s \geq t_{2}$. This means that $y(t)$ is eventually decreasing. If $y(t)$ is eventually decreasing, it follows from $y(t)>0$ that $\lim _{t \rightarrow \infty} y(t)=L$ exists and $|L|<\infty$. If there does not exist an $s \geq t_{1}$ such that $y^{\prime}(s) \leq 0$, then $y^{\prime}(s)>0$ for all $s \geq t_{1}$. This means $y(t)$ is eventually increasing. From (5), we have

$$
y(t)<y(s)+r(s) y^{\prime}(s) R(s, t) .
$$


Since $R_{0}<\infty$ and $y^{\prime}(t)>0$, we see that $y(t)$ is bounded. Therefore $\lim _{t \rightarrow \infty} y(t)=L$ exists and $|L|<\infty$.

Similarly, we can discuss the case when $x(t)$ is an eventually negative solution of (1). The proof is complete.

Lemma 6. Suppose that $(\mathrm{H})$ holds. If $x(t)$ is a nonoscillatory solution of (1) and $y(t)$ is defined by (2), then there exist two positive constants $a_{1}, a_{2}$ and $t_{1} \geq t_{0}$ such that either

$$
a_{1} R(t) \leq y(t) \leq a_{2} \quad \text { or } \quad-a_{2} \leq y(t) \leq-a_{1} R(t)
$$

for all $t \geq t_{1}$.

P r o of. Let $x(t)$ be an eventually positive solution of (1). By Lemma 1, $y(t)$ is eventually of constant sign. We have four cases to consider:

(i) $y(t)>0$ and $y^{\prime}(t)>0$ eventually;

(ii) $y(t)>0$ and $y^{\prime}(t)<0$ eventually;

(iii) $y(t)<0$ and $y^{\prime}(t)>0$ eventually;

(iv) $y(t)<0$ and $y^{\prime}(t)<0$ eventually.

We shall only consider cases (i) and (ii) in detail, since the other two cases are similar.

(i) If $y(t)$ is eventually increasing, then (6) holds. From Lemma 5, there exists a positive constant $a_{2}$ such that $y(t) \leq a_{2}$. Since we are assuming $y(t)$ is positive and increasing and since $R(t) \rightarrow 0$ as $t \rightarrow \infty$, there exists a positive constant $a_{1}$ and $t_{1} \geq t_{0}$ such that $y(t) \geq a_{1} R(t)$ for all $t \geq t_{1}$.

(ii) If $y(t)$ is eventually decreasing, then, from (6),

$$
y(s) \geq y(t)-r(s) y^{\prime}(s) R(s, t) .
$$

By Lemma $5, \lim _{t \rightarrow \infty} y(t)=L \geq 0$. Taking the limit as $t \rightarrow \infty$ on both sides of the last inequality, we can see that

$$
y(s) \geq L-r(s) y^{\prime}(s) R(s) .
$$

Since $r(t) y^{\prime}(t)$ is eventually decreasing, we can choose $t_{1}$ so large that $r\left(t_{1}\right) y^{\prime}\left(t_{1}\right)<0$; then $r(s) y^{\prime}(s) \leq r\left(t_{1}\right) y^{\prime}\left(t_{1}\right)=-a_{1}$ for all $s \geq t_{1}$. Therefore

$$
y(s) \geq a_{1} R(s)
$$

for $s \geq t_{1}$, where $a_{1}>0$ is independent of $s$. Changing $s$ to $t$, we can see that

for $t \geq t_{1}$.

$$
y(t) \geq a_{1} R(t), \quad a_{1}>0
$$

Similarly, we can prove the case when $x(t)$ is an eventually negative solution of (1). This completes the proof.

The following result is one of the main classification theorems. 
TheOREM 1. Suppose that $(\mathrm{H})$ holds and that $\lim _{t \rightarrow \infty} p(t)=p_{0}$, where $0 \leq p_{0}<1$. Then any nonoscillatory solution of (1) must belong to one of the following four types:

$$
\begin{aligned}
S(b, a, c): & x(t) \rightarrow b=\frac{a}{1-p_{0}} \neq 0, \quad y(t) \rightarrow a \neq 0, \\
& r(t) y^{\prime}(t) \rightarrow c \quad(t \rightarrow \infty), \\
S(b, a, \infty): & x(t) \rightarrow b=\frac{a}{1-p_{0}} \neq 0, \quad y(t) \rightarrow a \neq 0, \\
& r(t) y^{\prime}(t) \rightarrow \pm \infty \quad(t \rightarrow \infty), \\
S(0,0, c): \quad & x(t) \rightarrow 0, \quad y(t) \rightarrow 0, \quad r(t) y^{\prime}(t) \rightarrow c \neq 0 \quad(t \rightarrow \infty), \\
S(0,0, \infty): \quad & x(t) \rightarrow 0, \quad y(t) \rightarrow 0, \quad r(t) y^{\prime}(t) \rightarrow \pm \infty \quad(t \rightarrow \infty),
\end{aligned}
$$

where $a, b, c$ are some finite constants.

P r o o f. Suppose that $x(t)$ is a nonoscillatory solution of (1). By Lemmas 1 and $5, y(t)$ is eventually of constant sign and $\lim _{t \rightarrow \infty} y(t)=L$, where $L$ is a finite constant. So there are only two possibilities: $\lim _{t \rightarrow \infty} y(t)=a \neq 0$ or $\lim _{t \rightarrow \infty} y(t)=0$, where $a$ is a finite constant. According to Lemma 4, $\lim _{t \rightarrow \infty} x(t)=b \neq 0$ or $\lim _{t \rightarrow \infty} x(t)=0$.

In addition, by our assumption that $x f(t, x)>0$ for $x \neq 0$ we see from (1) that $r(t) y^{\prime}(t)$ is eventually decreasing or increasing. Again there are only two possibilities: $\lim _{t \rightarrow \infty} r(t) y^{\prime}(t)=c$ or $\lim _{t \rightarrow \infty} r(t) y^{\prime}(t)= \pm \infty$, where $c$ is a finite constant.

From the above discussion, we see that $x(t)$ must belong to one of the four types as stated, except that we have not yet shown that for the case $S(0,0, c)$, the constant $c \neq 0$. We do this next.

Suppose $x(t)$ is a nonoscillatory solution of (1) which belongs to $S(0,0, c)$, that is, $\lim _{t \rightarrow \infty} x(t)=0, \lim _{t \rightarrow \infty} y(t)=0$, and $\lim _{t \rightarrow \infty} r(t) y^{\prime}(t)=c$. Then $c \neq 0$. In fact, consider functions $y(t)$ and $R(t)$. From our assumption, it is easy to see that

$$
\lim _{t \rightarrow \infty} y(t)=0, \quad \lim _{t \rightarrow \infty} R(t)=0, \quad R^{\prime}(t)<0,
$$

and

$$
\lim _{t \rightarrow \infty} \frac{y^{\prime}(t)}{R^{\prime}(t)}=\lim _{t \rightarrow \infty}-r(t) y^{\prime}(t)=-c .
$$

By l'Hopital's theorem, $\lim _{t \rightarrow \infty}(y(t) / R(t))$ exists, and

$$
\lim _{t \rightarrow \infty} \frac{y(t)}{R(t)}=\lim _{t \rightarrow \infty} \frac{y^{\prime}(t)}{R^{\prime}(t)}=-c .
$$

If $y(t)$ is eventually positive, then, by Lemma 6 , there exist two positive 
constants $a_{1}$ and $a_{2}$ such that $a_{1} R(t) \leq y(t) \leq a_{2}$. Thus $y(t) / R(t) \geq a_{1}$; that is, $a_{1} \leq-c$. It follows that $c \neq 0$. If $y(t)$ is eventually negative, then by Lemma 6 , there exist two positive constants $a_{1}$ and $a_{2}$ such that $-a_{2} \leq$ $y(t) \leq-a_{1} R(t)$. Thus $y(t) / R(t) \leq-a_{1}$, which means $-c \leq-a_{1}$. Once more it follows that $c \neq 0$, which completes the proof.

Next we derive two existence theorems.

Theorem 2. Assume that $(\mathrm{H})$ holds and $\lim _{t \rightarrow \infty}(R(t-\tau) / R(t))=1$. A necessary and sufficient condition for (1) to have a nonoscillatory solution $x(t)$ which belongs to $S(0,0, c)$ is that

$$
\int_{t_{0}}^{\infty}|f(t, \lambda R(t-\delta))| d t<\infty
$$

for some $\lambda \neq 0$.

Proof. Necessity. Let $x(t)$ be any nonoscillatory positive solution of (1) which belongs to $S(0,0, c)$, i.e., $\lim _{t \rightarrow \infty} x(t)=0, \lim _{t \rightarrow \infty} y(t)=0$ and $\lim _{t \rightarrow \infty} r(t) y^{\prime}(t)=c \neq 0$. Without loss of generality we may assume that $c<0$. Since $r(t) y^{\prime}(t)$ is monotone, there exist two positive constants $\lambda_{1}, \lambda_{2}$ and $t_{1} \geq t_{0}$ such that $-\lambda_{1} \leq r(t) y^{\prime}(t) \leq-\lambda_{2}$ for $t \geq t_{1}$. It follows that

$$
-\lambda_{1} R(t, s) \leq y(s)-y(t) \leq-\lambda_{2} R(t, s)
$$

for $s>t, t \geq t_{1}$. Let $s \rightarrow \infty$; then $-\lambda_{1} R(t) \leq-y(t) \leq-\lambda_{2} R(t)$. That is, $\lambda_{2} R(t) \leq y(t) \leq \lambda_{1} R(t)$. On the other hand, by (1),

$$
r(t) y^{\prime}(t)=r\left(t_{1}\right) y^{\prime}\left(t_{1}\right)-\int_{t_{1}}^{t} f(s, x(s-\delta)) d s .
$$

Since $\lim _{t \rightarrow \infty} r(t) y^{\prime}(t)=c<0$, we have

$$
\int_{t_{1}}^{\infty}|f(s, x(s-\delta))| d s<\infty .
$$

Furthermore, since $(\mathrm{H})$ holds, by $y(t) \leq x(t)$, we have

$$
f(s, x(s-\delta)) \geq f(s, y(s-\delta)) \geq f\left(s, \lambda_{2} R(s-\delta)\right) .
$$

This means that

$$
\int_{t_{1}}^{\infty}\left|f\left(s, \lambda_{2} R(s-\delta)\right)\right| d s<\infty
$$

Sufficiency. Suppose that (7) holds for $\lambda>0$. A similar argument can be applied if $\lambda<0$. Since $\lim _{t \rightarrow \infty}(R(t-\tau) / R(t))=1$, we may choose $p<A<1$ 
and $t_{1} \geq t_{0}$ such that $p(t) R(t-\tau) / R(t) \leq A$ and

$$
\int_{t_{1}}^{\infty} f(s, \lambda R(s-\tau)) d s<a(1-A),
$$

where $a=\lambda / 2$. Consider the equation

$$
\begin{aligned}
x(t)= & p(t) x(t-\tau)+R(t)\left[(1-A) a+\int_{t_{1}}^{t} f(s, x(s-\delta)) d s\right] \\
& +\int_{t}^{\infty} R(s) f(s, x(s-\delta)) d s
\end{aligned}
$$

for $t \geq t_{1}+\max \{\delta, \tau\}$. It is easy to see that a solution of (9) must also be a solution of (1). We shall show that (9) has a nonoscillatory solution $x(t)$ which belongs to $S(0,0, c)$ by means of the method of successive approximations. Consider the sequence $\left\{x^{k}(t)\right\}$ of successive approximating sequences defined as follows:

$$
\begin{aligned}
x^{1}(t) & =0, & & t_{0} \leq t \leq t_{1}, \\
x^{i+1}(t) & =\left(F x^{i}\right)(t), & & t \geq t_{1}, i=1,2, \ldots,
\end{aligned}
$$

where $F$ is defined by

$$
\begin{aligned}
(F x)(t)= & p(t) x(t-\tau)+R(t)\left[(1-A) a+\int_{t_{1}}^{t} f(s, x(s-\delta)) d s\right] \\
& +\int_{t}^{\infty} R(s) f(s, x(s-\delta)) d s
\end{aligned}
$$

for $t \geq t_{1}+\max \{\delta, \tau\}$, and $(F x)(t)=(F x)\left(t_{1}+\max \{\delta, \tau\}\right)$ for $t_{1} \leq t \leq$ $t_{1}+\max \{\delta, \tau\}$. In view of $\left(\mathrm{H}_{1}\right)$, it is easy to see that

$$
0 \leq x^{i}(t) \leq x^{i+1}(t), \quad t \geq t_{1}, i=1,2, \ldots
$$

On the other hand,

$$
x^{2}(t)=\left(F x^{1}\right)(t)=a(1-A) R(t) \leq 2 a R(t), \quad t \geq t_{1},
$$

and inductively, we have

$$
\begin{aligned}
\left(F x^{i}\right)(t) & \leq 2 p(t) a R(t-\tau)+(1-A) a R(t)+(1-A) a R(t) \\
& \leq 2 A a R(t)+2(1-A) a R(t)=2 a R(t)
\end{aligned}
$$

for $i \geq 2$. Thus, by means of Lebesgue's dominated convergence theorem, we see that $F x=x$. Furthermore, it is clear that $x(t)$, and hence its associated function $y(t)$, converges to zero (since $R(t)$ does). Finally, in view of (9), we 
see that

$$
\begin{aligned}
-(1-A) a>r(t) y^{\prime}(t) & =-(1-A) a-\int_{t_{1}}^{t} f(s, x(s-\delta)) d s \\
& >-(1-A) a-\int_{t_{1}}^{t} f(s, \lambda R(s-\delta)) d s \\
& >-(1-A) a-(1-A) a=-2(1-A) a,
\end{aligned}
$$

which implies $\lim _{t \rightarrow \infty} r(t) y^{\prime}(t)=c \neq 0$ as required. The proof is complete.

Theorem 3. Assume that $(\mathrm{H})$ holds and that $\lim _{t \rightarrow \infty} p(t)=p_{0}$, where $0 \leq p_{0}<1$. A necessary and sufficient condition for (1) to have a nonoscillatory solution $x(t)$ which belongs to $S(b, a, c)$ or $S(b, a, \infty)$ is that

$$
\int_{t_{2}}^{\infty} \frac{1}{r(s)} \int_{t_{1}}^{s}|f(u, \lambda)| d u d s<\infty
$$

for some $\lambda \neq 0$ and $t_{2} \geq t_{1} \geq t_{0}$.

Proof. Necessity. Let $x(t)$ be any nonoscillatory positive solution of (1) such that $\lim _{t \rightarrow \infty} x(t)=b>0$. By (2), we have $\lim _{t \rightarrow \infty} y(t)=a=$ $b\left(1-p_{0}\right)>0$, which means that $y(t)$ is eventually positive and monotonically tends to $a$. Thus there exist two positive constants $c_{1}, c_{2}$ and $t_{1} \geq t_{0}$ such that $c_{1} \leq y(t) \leq c_{2}$ for $t \geq t_{1}$. It follows from (1) that

$$
y(t)=y(s)+r\left(t_{1}\right) y^{\prime}\left(t_{1}\right) \int_{s}^{t} \frac{1}{r(u)} d u-\int_{s}^{t} \frac{1}{r(u)} \int_{t_{1}}^{u} f(v, x(v-\delta)) d v d u .
$$

Taking the limit as $t \rightarrow \infty$ on both sides of the last equality, we obtain

$$
a=y(s)+r\left(t_{1}\right) y^{\prime}\left(t_{1}\right) \int_{s}^{\infty} \frac{1}{r(u)} d u-\int_{s}^{\infty} \frac{1}{r(u)} \int_{t_{1}}^{u} f(v, x(v-\delta)) d v d u .
$$

This means that

$$
0 \leq \int_{s}^{\infty} \frac{1}{r(u)} \int_{t_{1}}^{u} f(v, x(v-\delta)) d v d u<\infty .
$$

By $(\mathrm{H})$, we have $f\left(t, c_{1}\right) \leq f(t, y(t-\delta)) \leq f(t, x(t-\delta))$. It follows from (12) that

$$
\int_{s}^{\infty} \frac{1}{r(u)} \int_{t_{1}}^{u} f\left(v, c_{1}\right) d v d u<\infty
$$

Sufficiency. Suppose that (11) holds for $\lambda>0$. A similar argument can 
be applied if $\lambda<0$. Choose $t_{1} \geq t_{0}$ so large that

$$
\int_{s}^{\infty} \frac{1}{r(u)} \int_{t_{1}}^{u} f(v, \lambda) d v d u<(1-p) a,
$$

where $a=\lambda / 2$. Consider the equation

$$
x(t)=p(t) x(t-\tau)+(1-p) a+\int_{t}^{\infty} \frac{1}{r(u)} \int_{t_{1}}^{u} f(v, x(v-\delta)) d v d u .
$$

It is easy to verify that a solution of (14) must also be a solution of (1). Consider the Banach space $\Phi$ of all bounded real functions $x(t)$ with norm $\sup _{t \geq t_{1}}|x(t)|$, endowed with the usual pointwise ordering $\leq$ : for $x, y \in \Phi$, $x \leq y$ is equivalent to $x(t) \leq y(t)$ for $t \geq t_{1}$. Then $\Phi$ is partially ordered. Define a subset $\Omega$ of $\Phi$ as follows:

$$
\Omega=\left\{x \in \Phi:(1-p) a \leq x(t) \leq 2 a, t \geq t_{1}\right\} .
$$

For any subset $B \subset \Omega$, it is obvious that $\inf B \in \Omega$ and $\sup B \in \Omega$. We also define an operator $F: \Omega \rightarrow \Phi$ as

$$
(F x)(t)=p(t) x(t-\tau)+(1-p) a+\int_{t}^{\infty} \frac{1}{r(u)} \int_{t_{1}}^{u} f(v, x(v-\delta)) d v d u, \quad t \geq t_{1} .
$$

The mapping $F$ satisfies the assumptions of Knaster's fixed point theorem [1]. Namely, it satisfies the following:

(i) $F$ maps $\Omega$ into itself. Indeed, if $x \in \Omega$, by (13), we have

$$
(1-p) a \leq(F x)(t) \leq 2 p(t) a+(1-p) a+(1-p) a \leq 2 p a+2(1-p) a=2 a .
$$

(ii) By $(\mathrm{H}), F$ is nondecreasing. That is, for any $x, y \in \Omega, x \leq y$ implies that $F x \leq F y$.

By Knaster's fixed point theorem [1], there exists an $x \in \Omega$ such that $F x=x$, that is, $x(t)$ is a nonoscillatory solution of (1) which belongs to $S(b, a, c)$ or $S(b, a, \infty)$. Note that $b \neq 0$. Since $x \in \Omega$ and by (2) and Lemma $4, \lim _{t \rightarrow \infty} y(t)=a$ and $\lim _{t \rightarrow \infty} x(t)=a /\left(1-p_{0}\right)=b \neq 0$ must exist. This completes the proof.

4. The case $R_{0}=\infty$. When $R_{0}=\infty$, the asymptotic behaviors of the nonoscillatory solutions of (1) are quite different from those obtained in Section 3.

Lemma 7. Suppose that $(\mathrm{H})$ holds. If $x(t)$ is an eventually positive (negative) solution of (1) and $\lim \sup _{t \rightarrow \infty} x(t)>0$ (resp. $\lim \sup _{t \rightarrow \infty}(-x(t))$ $>0)$, then there exist two positive constants $a_{1}, a_{2}$ and $t_{1} \geq t_{0}$ such that the function $y(t)$ defined by (2) is monotonically increasing (decreasing) and 


$$
a_{1} \leq y(t) \leq a_{2} R\left(t_{1}, t\right) \quad\left(\text { resp. }-a_{2} R\left(t_{1}, t\right) \leq y(t) \leq-a_{1}\right)
$$

for all $t \geq t_{1}$.

Proof. Let $x(t)$ be an eventually positive solution of (1) and $\lim \sup _{t \rightarrow \infty} x(t)>0$. Then, by part (ii) of Lemma 2, we have $y(t)>0$ eventually. Thus, there exists $s \geq t_{0}$ such that $x(t)>0$ and $y(t)>0$ for all $t \geq s$. It follows from (1) that $\left(r(t) y^{\prime}(t)\right)^{\prime}<0$ and thus $r(t) y^{\prime}(t)$ is eventually decreasing. By Lemma 3, and in view of $R_{0}=\infty$, we have $y^{\prime}(t)>0$ eventually. Thus, $y(t)$ is eventually increasing and $r(t) y^{\prime}(t)$ is positive. By (3) we then see that (15) holds. This completes the proof.

Theorem 4. Suppose that $(\mathrm{H})$ holds and $\lim _{t \rightarrow \infty} p(t)=p_{0}$, where $0 \leq$ $p_{0}<1$. Then any nonoscillatory solution of (1) must belong to one of the following five types:

$$
\begin{aligned}
S(0,0,0): & x(t) \rightarrow 0, \quad y(t) \rightarrow 0, \quad r(t) y^{\prime}(t) \rightarrow 0 \quad(t \rightarrow \infty), \\
S(0,0, c): & x(t) \rightarrow 0, \quad y(t) \rightarrow 0, \quad r(t) y^{\prime}(t) \rightarrow c \neq 0 \quad(t \rightarrow \infty), \\
S(b, a, 0): & x(t) \rightarrow b=\frac{a}{1-p_{0}} \neq 0, \quad y(t) \rightarrow a \neq 0, \\
& r(t) y^{\prime}(t) \rightarrow 0 \quad(t \rightarrow \infty), \\
S(\infty, \infty, c): & x(t) \rightarrow \infty, \quad y(t) \rightarrow \infty, \quad r(t) y^{\prime}(t) \rightarrow c \neq 0 \quad(t \rightarrow \infty), \\
S(\infty, \infty, 0): & x(t) \rightarrow \infty, \quad y(t) \rightarrow \infty, \quad r(t) y^{\prime}(t) \rightarrow 0 \quad(t \rightarrow \infty),
\end{aligned}
$$

where $a, b, c$ are some finite constants.

P r o o f. For any nonoscillatory solution of (1), without loss of generality, we may suppose that $x(t)$ is an eventually positive solution. If $\lim _{t \rightarrow \infty} x(t)$ $=0$, then, by part (i) of Lemma $2, \lim _{t \rightarrow \infty} y(t)=0$ and $y^{\prime}(t)>0$ for all large $t$. Since $\left(r(t) y^{\prime}(t)\right)^{\prime}<0$ for all large $t$, we have

$$
\lim _{t \rightarrow \infty} r(t) y^{\prime}(t)=c \geq 0 .
$$

If $\limsup _{t \rightarrow \infty} x(t)>0$, in view of part (ii) of Lemma 2, we have $y(t)>0$ for all large $t$. By Lemma 7, we know that $y(t)$ is eventually increasing, and $r(t) y^{\prime}(t)$ is positive and decreasing. Thus there exist only the following possibilities:

$$
\lim _{t \rightarrow \infty} y(t)=a, \quad 0<a<\infty, \quad \text { or } \quad \lim _{t \rightarrow \infty} y(t)=\infty,
$$

and

$$
\lim _{t \rightarrow \infty} r(t) y^{\prime}(t)=c \geq 0 .
$$

Since $r(t) y^{\prime}(t)$ is eventually decreasing and $\lim _{t \rightarrow \infty} r(t) y^{\prime}(t)=c$, we have

$$
r(t) y^{\prime}(t) \geq c, \quad \text { i.e., } \quad y^{\prime}(t) \geq \frac{c}{r(t)}
$$


for all large $t$. Integrating the inequality, we obtain

$$
y(t) \geq y(s)+c \int_{s}^{t} \frac{1}{r(u)} d u .
$$

If $c>0$ and $R_{0}=\infty$, it follows that

$$
\lim _{t \rightarrow \infty} y(t)=\infty .
$$

By Lemma 4, we have $\lim _{t \rightarrow \infty} x(t)=\infty$. If $c=0$ and $\lim _{t \rightarrow \infty} y(t)=a$ $($ resp. $\infty)$, then, by Lemma 4 , it follows that $\lim _{t \rightarrow \infty} x(t)=b=a /\left(1-p_{0}\right)$ $($ resp. $\infty)$. Therefore $x(t)$ must belong to one of the five types as stated. The proof is complete.

Next we derive several existence criteria for the nonoscillatory solutions of $(1)$.

Theorem 5. Assume that $(\mathrm{H})$ holds and $\lim _{t \rightarrow \infty} p(t)=p_{0}$, where $0 \leq$ $p_{0}<1$. A necessary and sufficient condition for (1) to have a nonoscillatory solution which belongs to $S(b, a, 0)$ is that

$$
\int_{t_{0}}^{\infty} \frac{1}{r(u)} \int_{u}^{\infty}|f(v, \lambda)| d v d u<\infty
$$

for some $\lambda \neq 0$.

Proof. Necessity. Let $x(t)$ be a nonoscillatory solution of (1) which belongs to $S(b, a, 0)$, i.e.,

$$
\lim _{t \rightarrow \infty} x(t)=b, \quad \lim _{t \rightarrow \infty} y(t)=a, \quad \text { and } \quad \lim _{t \rightarrow \infty} r(t) y^{\prime}(t)=0 .
$$

Without loss of generality, we may suppose that $b>0$. By (2), it follows that $\lim _{t \rightarrow \infty} y(t)=a=b\left(1-p_{0}\right)>0$. Then there exist two positive constants $c_{1}, c_{2}$ and $t_{1} \geq t_{0}$ such that

$$
c_{1} \leq y(t) \leq c_{2} \quad \text { for } t \geq t_{1} .
$$

On the other hand, by (1),

$$
r(t) y^{\prime}(t)=r(s) y^{\prime}(s)-\int_{s}^{t} f(u, x(u-\delta)) d u
$$

for $t \geq s \geq t_{1}$. After taking the limit as $t \rightarrow \infty$ on both sides of (19) and using $\lim _{t \rightarrow \infty} r(t) y^{\prime}(t)=0$, we obtain

$$
r(s) y^{\prime}(s)=\int_{s}^{\infty} f(u, x(u-\delta)) d u
$$


for $s \geq t_{1}$. Now it follows from (20) that

$$
y(s)=y\left(t_{1}\right)+\int_{t_{1}}^{s} \frac{1}{r(u)} \int_{u}^{\infty} f(v, x(v-\delta)) d v d u
$$

for $s \geq t_{1}$. Let $s \rightarrow \infty$. Then

$$
a=y\left(t_{1}\right)+\int_{t_{1}}^{\infty} \frac{1}{r(u)} \int_{u}^{\infty} f(v, x(v-\delta)) d v d u
$$

Therefore

$$
\int_{t_{1}}^{\infty} \frac{1}{r(u)} \int_{u}^{\infty} f(v, x(v-\delta)) d v d u<\infty
$$

By $(\mathrm{H}), f\left(t, c_{1}\right) \leq f(t, y(t-\delta)) \leq f(t, x(t-\delta))$, so (17) holds.

Sufficiency. Without loss of generality we may assume that (17) holds for $\lambda>0$. Then there exists $t_{1} \geq t_{0}$ such that

$$
\int_{t_{1}}^{\infty} \frac{1}{r(u)} \int_{u}^{\infty} f(v, \lambda) d v d u<(1-p) a
$$

where $a=\lambda / 2$. Now consider the equation

$$
x(t)=p(t) x(t-\tau)+(1-p) a+\int_{t_{1}}^{t} \frac{1}{r(u)} \int_{u}^{\infty} f(v, x(v-\delta)) d v d u
$$

for $t \geq t_{1}$. Consider the Banach space $\Phi$ of all bounded real functions $x(t)$ with norm $\sup _{t>t_{1}}|x(t)|$, endowed with the usual pointwise ordering $\leq$ : for $x, y \in \Phi, x \leq y$ is equivalent to $x(t) \leq y(t)$ for $t \geq t_{1}$. Then $\Phi$ is partially ordered. Define a subset $\Omega$ of $\Phi$ as follows:

$$
\Omega=\left\{x \in \Phi:(1-p) a \leq x(t) \leq 2 a, t \geq t_{1}\right\} .
$$

If $x(t) \in \Phi$, let also

$$
\begin{aligned}
(F x)(t)= & p(t) x(t-\tau)+(1-p) a \\
& +\int_{t_{1}}^{t} \frac{1}{r(u)} \int_{u}^{\infty} f(v, x(v-\delta)) d v d u, \quad t \geq t_{1} .
\end{aligned}
$$

Then, by using Knaster's fixed point theorem and Lemma 7, we can show that there exists a nonoscillatory solution of (23), and thus of (1), which belongs to $S(b, a, 0)$. We omit the details. The proof is complete. 
TheOREM 6. Assume that $(\mathrm{H})$ holds. A necessary and sufficient condition for (1) to have a nonoscillatory solution which belongs to $S(\infty, \infty, c)$ is that

$$
\int_{t_{1}}^{\infty}\left|f\left(u, \lambda R\left(t_{1}, u-\delta\right)\right)\right| d u<\infty
$$

for some $\lambda \neq 0$ and $t_{1} \geq t_{0}$.

P r o of. Necessity. Suppose that $x(t)$ is an eventually positive solution of (1) which belongs to $S(\infty, \infty, c)$. That is,

$$
\lim _{t \rightarrow \infty} x(t)=\infty, \quad \lim _{t \rightarrow \infty} y(t)=\infty, \quad \lim _{t \rightarrow \infty} r(t) y^{\prime}(t)=c>0 .
$$

Then it follows from (15) and (16) that there exist two positive constants $c_{1}, c_{2}$ and $t_{1} \geq t_{0}$ such that

$$
c_{1} R\left(t_{1}, t\right) \leq y(t) \leq c_{2} R\left(t_{1}, t\right)
$$

for $t \geq t_{1}$. On the other hand, by (1),

$$
r(t) y^{\prime}(t)=r\left(t_{1}\right) y^{\prime}\left(t_{1}\right)-\int_{t_{1}}^{t} f(u, x(u-\delta)) d u .
$$

Let $t \rightarrow \infty$ on both sides of (27); we obtain

$$
\int_{t_{1}}^{\infty}|f(u, x(u-\delta))| d u<\infty .
$$

Since $(\mathrm{H})$ holds, $f\left(t, c_{1} R\left(t_{1}, t-\delta\right)\right) \leq f(t, y(t-\delta)) \leq f(t, x(t-\delta))$ and we can conclude from (28) that (24) holds for some $\lambda \neq 0$ and $t_{1} \geq t_{0}$.

Sufficiency. Without loss of generality, we may assume that (24) holds for $\lambda>0$ and $t_{1} \geq t_{0}$. Then there exists $t_{2} \geq t_{1}$ such that

$$
\int_{t_{2}}^{\infty} f\left(u, \lambda R\left(t_{1}, u-\delta\right)\right) d u<(1-p) a,
$$

where $a=\lambda / 2$. Now consider the equation

$$
\begin{aligned}
(29) x(t)= & p(t) x(t-\tau)+(1-p) a R\left(t_{1}, t\right)+\int_{t_{2}}^{t} R\left(t_{2}, u\right) f(u, x(u-\delta)) d u \\
& +R\left(t_{1}, t\right) \int_{t}^{\infty} f(u, x(u-\delta)) d u
\end{aligned}
$$

for $t \geq t_{2}$. We introduce the Banach space $\Phi$ of all bounded real functions $x(t)$ which satisfy

$$
\sup _{t \geq t_{2}} \frac{|x(t)|}{R\left(t_{1}, t\right)}<\infty
$$


with the norm

$$
\|x\|=\sup _{t \geq t_{2}} \frac{|x(t)|}{R\left(t_{1}, t\right)} .
$$

$\Phi$ is considered to be endowed with the usual pointwise ordering $\leq$ : for $x, y \in \Phi, x \leq y$ is equivalent to $x(t) \leq y(t)$ for $t \geq t_{2}$. Then $\Phi$ is partially ordered. Define a subset $\Omega$ of $\Phi$ and an operator $F: \Omega \rightarrow \Phi$ as follows:

$$
\Omega=\left\{x \in \Phi:(1-p) a R\left(t_{1}, t\right) \leq x(t) \leq 2 a R\left(t_{1}, t\right), t \geq t_{2}\right\},
$$

and for $x(t) \in \Phi$,

$$
\begin{aligned}
(F x)(t)= & p(t) x(t-\tau)+(1-p) a R\left(t_{1}, t\right) \\
& +\int_{t_{2}}^{t} R\left(t_{2}, u\right) f(u, x(u-\delta)) d u \\
& +R\left(t_{1}, t\right) \int_{t}^{\infty} f(u, x(u-\delta)) d u, \quad t \geq t_{2} .
\end{aligned}
$$

Similarly to the proof of Theorem 3, we can show that there exists a nonoscillatory solution of (29), and hence also of (1), which belongs to $S(\infty, \infty, c)$. This completes the proof.

TheOREM 7. Assume that $(\mathrm{H})$ holds. A sufficient condition for (1) to have a nonoscillatory solution which belongs to $S(\infty, \infty, 0)$ is that

$$
\int_{t_{0}}^{\infty} R\left(t_{0}, u\right)\left|f\left(u, \lambda_{1} R\left(t_{1}, u-\delta\right)\right)\right| d u=\infty
$$

and

$$
\int_{t_{0}}^{\infty}\left|f\left(u, \lambda_{2} R\left(t_{1}, u-\delta\right)\right)\right| d u<\infty
$$

for some $\lambda_{1}, \lambda_{2}$ such that $\lambda_{1} \lambda_{2}>0$ and $t_{1} \geq t_{0}$.

Pro of. Without loss of generality, we may assume $\lambda_{1}>0, \lambda_{2}>0$. Take $t_{2} \geq t_{1}$ such that for $t \geq t_{2}$,

$$
\frac{\lambda_{1}}{R\left(t_{1}, t\right) \lambda_{2}}+p(t)+\frac{1}{\lambda_{2}} \int_{t_{2}}^{\infty} f\left(u, \lambda_{2} R\left(t_{1}, u-\delta\right)\right) d u<1 .
$$


Define an operator $F$ as

$$
\begin{aligned}
(F x)(t)= & \frac{\lambda_{1}}{R\left(t_{1}, t\right)}+\frac{R\left(t_{1}, t-\tau\right)}{R\left(t_{1}, t\right)} p(t) x(t-\tau) \\
& +\frac{1}{R\left(t_{1}, t\right)} \int_{t_{1}}^{t} R\left(t_{1}, u\right) f\left(u, R\left(t_{1}, u-\delta\right) x(u-\delta)\right) d u \\
& +\int_{t}^{\infty} f\left(u, R\left(t_{1}, u-\delta\right) x(u-\delta)\right) d u, \quad t \geq t_{2},
\end{aligned}
$$

and

$$
(F x)(t)=F x\left(t_{2}\right), \quad t_{1} \leq t \leq t_{2},
$$

and let $x^{1}(t)=0, x^{i+1}(t)=\left(F x^{i}\right)(t), t \geq t_{1}, i=1,2, \ldots$ By $(\mathrm{H})$ and induction, it is easy to see that $0 \leq x^{i}(t) \leq x^{i+1}(t), t \geq t_{1}, i=1,2, \ldots$ On the other hand, $x^{2}(t) \leq \lambda_{2}, t \geq t_{1}$. It follows from (32) that

$$
x^{i+1}(t) \leq \lambda_{2}\left[\frac{\lambda_{1}}{R\left(t_{1}, t\right) \lambda_{2}}+p(t)+\frac{1}{\lambda_{2}} \int_{t_{2}}^{\infty} f\left(u, \lambda_{2} R\left(t_{1}, u-\delta\right)\right) d u\right] \leq \lambda_{2} .
$$

By induction, we have $x^{i}(t) \leq \lambda_{2}$ for $t \geq t_{1}, i=1,2, \ldots$ Let

$$
\lim _{i \rightarrow \infty} x^{i}(t)=x^{*}(t), \quad t \geq t_{2} .
$$

Using Lebesgue's dominated convergence theorem, we get

$$
x^{*}(t)=\left(F x^{*}\right)(t), \quad t \geq t_{1} .
$$

By (32) and (33), it is easy to see that

$$
\frac{\lambda_{1}}{R\left(t_{1}, t\right)} \leq x^{*}(t) \leq \lambda_{2} .
$$

Set

$$
z(t)=R\left(t_{1}, t\right) x^{*}(t), \quad t \geq t_{1} .
$$

Then we have $\lambda_{1} \leq z(t) \leq \lambda_{2} R\left(t_{1}, t\right)$ and

$$
\begin{aligned}
z(t)= & \lambda_{1}+p(t) z(t-\tau)+\int_{t_{1}}^{t} R\left(t_{1}, u\right) f(u, z(u-\delta)) d u \\
& +R\left(t_{1}, t\right) \int_{t}^{\infty} f(u, z(u-\delta)) d u, \quad t \geq t_{2} .
\end{aligned}
$$

Again set

$$
w(t)=z(t)-p(t) z(t-\tau)
$$


then, by (30), we have $\lim _{t \rightarrow \infty} w(t)=\infty$. From part (ii) of Lemma 2 , it follows that $\lim _{t \rightarrow \infty} z(t)=\infty$. Since

$$
r(t) w^{\prime}(t)=\int_{t}^{\infty} f(u, z(u-\delta)) d u
$$

it follows that $\lim _{t \rightarrow \infty} r(t) w^{\prime}(t)=0$. This means $z(t)$ is a positive solution of (1) that belongs to $S(\infty, \infty, 0)$, which completes the proof.

Theorem 8. Assume that $(\mathrm{H})$ holds, that $|p(t)-p(s)| \leq K|t-s|$ and that $r(t)$ is nondecreasing. Furthermore, suppose that there exist $K_{1}>K_{2}>0$ such that

$$
p(t) e^{K_{1} \tau}>1 \geq p(t) e^{K_{2} \tau},
$$

and for large $t_{1}$ and $t \geq t_{1}$,

$$
\left(p(t) e^{K_{1} \tau}-1\right) e^{-K_{1} t} \geq \int_{t}^{\infty} \frac{1}{r(u)} \int_{u}^{\infty} f\left(v, e^{-K_{2}(v-\delta)}\right) d v d u .
$$

Then (1) has a nonoscillatory solution $x(t) \in S(0,0,0)$.

Proof. Set

$$
\begin{aligned}
& \Omega=\{x \in C[0, \infty): \\
& \left.\quad e^{-K_{1} t} \leq x(t) \leq e^{-K_{2} t},|x(t)-x(s)| \leq L|t-s|, t \geq s \geq t_{0}\right\},
\end{aligned}
$$

where $L \geq \max \left\{K, K_{1}\right\}$. It is easy to show that $\Omega$ is nonempty, bounded, convex and closed in $\Phi$ defined to be all bounded real functions $x(t)$ with norm $\sup _{t \geq t_{0}}|x(t)|$.

Define an operator $F$ on $\Omega$ as

$$
(F x)(t)=\left\{\begin{array}{l}
p(t) x(t-\tau)-\int_{t}^{\infty} \frac{1}{r(u)} \int_{u}^{\infty} f(v, x(v-\delta)) d v d u, \quad t \geq t_{2}, \\
\exp \left(\frac{\ln \left((F x)\left(t_{2}\right)\right)}{t_{2}} t\right), \quad t_{0} \leq t \leq t_{2}
\end{array}\right.
$$

where $t_{2} \geq t_{1}$, and for given $\alpha, p<\alpha<1$, let

$$
\frac{1}{r(t)} \int_{t_{2}}^{\infty} f\left(s, e^{-K_{2}(s-\delta)}\right) d s \leq(\alpha-p(t)) L, \quad t \geq t_{2},
$$

and

$$
\alpha+e^{-K_{2}(t-\tau)} \leq 1, \quad t \geq t_{2} .
$$

The mapping $F$ satisfies the assumptions of Schauder's fixed point theorem. Namely, it satisfies the following:

(i) $F$ maps $\Omega$ into $\Omega$. For any $x \in S$, by (34) and (35) we obtain $(F x)(t) \leq p(t) x(t-\tau) \leq p(t) \exp \left(-K_{2}(t-\tau)\right) \leq \exp \left(-K_{2} t\right)$, 


$$
\begin{aligned}
(F x)(t) \geq & p(t) \exp \left(-K_{1}(t-\tau)\right)-\int_{t}^{\infty} \frac{1}{r(u)} \int_{u}^{\infty} f\left(v, \exp \left(-K_{2}(v-\delta)\right)\right) d v d u \\
= & \exp \left(-K_{1} t\right)+\left(p(t) \exp \left(K_{1} \tau\right)-1\right) \exp \left(-K_{1} t\right) \\
& \quad-\int_{t}^{\infty} \frac{1}{r(u)} \int_{u}^{\infty} f\left(v, \exp \left(-K_{2}(v-\delta)\right)\right) d v d u \\
\geq & \exp \left(-K_{1} t\right)
\end{aligned}
$$

and so

$$
K_{2} \leq \frac{-\ln \left((F x)\left(t_{2}\right)\right)}{t_{2}} \leq K_{1} .
$$

For any $s \geq t \geq t_{2}$, by (37) and (38) we get

$$
\begin{aligned}
|(F x)(s)-(F x)(t)| \leq & {\left[\left(p(s)+\exp \left(-K_{2}(t-\tau)\right)\right) L\right.} \\
& \left.+\frac{1}{r(t)} \int_{t}^{\infty} f\left(u, \exp \left(-K_{2}(u-\delta)\right)\right) d u\right]|s-t| \\
\leq & {\left[\exp \left(-K_{2}(t-\tau)\right)+\alpha\right] L|s-t| \leq L|s-t| }
\end{aligned}
$$

and for $t_{0} \leq t \leq s \leq t_{2}$,

$|(F x)(s)-(F x)(t)|=\left|\exp \left(\frac{\ln \left((F x)\left(t_{2}\right)\right)}{t_{2}} s\right)-\exp \left(\frac{\ln \left((F x)\left(t_{2}\right)\right)}{t_{2}} t\right)\right| \leq L|s-t|$.

Hence, $F$ maps $\Omega$ into $\Omega$.

(ii) $F$ is continuous. Let $x^{i}(t) \in \Omega$ such that

$$
\lim _{i \rightarrow \infty}\left\|x^{i}-x\right\|=0 .
$$

Since $\Omega$ is closed, $x \in \Omega$. Then, by (36), we get

$$
\begin{aligned}
& \left|\left(F x^{i}\right)(t)-(F x)(t)\right| \\
& \quad \leq p(t)\left\|x^{i}-x\right\|+\int_{t_{2}}^{\infty} \frac{1}{r(u)} \int_{u}^{\infty}\left|f\left(v, x^{i}(v-\delta)\right)-f(v, x(v-\delta))\right| d v d u \\
& \quad \leq p\left\|x^{i}-x\right\|+\int_{t_{2}}^{\infty} \frac{1}{r(u)} \int_{u}^{\infty}\left|f\left(v, x^{i}(v-\delta)\right)-f(v, x(v-\delta))\right| d v d u .
\end{aligned}
$$

By the continuity of $f$ and Lebesgue's dominated convergence theorem, it follows that

$$
\lim _{i \rightarrow \infty} \sup _{t \geq t_{2}}\left|\left(F x^{i}\right)(t)-(F x)(t)\right|=0 .
$$


We can easily show that

$$
\sup _{t_{0} \leq t \leq t_{2}}\left|\left(F x^{i}\right)(t)-(F x)(t)\right| \leq\left|\ln \left(\left(F x^{i}\right)\left(t_{2}\right)\right)-\ln \left((F x)\left(t_{2}\right)\right)\right| .
$$

Using (40) and (41), it is easy to show that

$$
\lim _{i \rightarrow \infty}\left\|F x^{i}-F x\right\|=0 .
$$

(iii) $F(\Omega)$ is precompact. Let $x \in \Omega$ and $s, t \geq t_{2}$. Then by (37) we have, for $s>t$,

$$
\begin{aligned}
|(F x)(t)-(F x)(s)|= & \mid p(t) x(t-\tau)-p(s) x(s-\tau) \\
& +\int_{s}^{\infty} \frac{1}{r(u)} \int_{u}^{\infty} f(v, x(v-\delta)) d v d u \\
& \quad-\int_{t}^{\infty} \frac{1}{r(u)} \int_{u}^{\infty} f(v, x(v-\delta)) d v d u \mid \\
\leq & |p(t) x(t-\tau)-p(s) x(s-\tau)| \\
& +\left|\int_{s}^{t} \frac{1}{r(u)} \int_{u}^{\infty} f(s, x(v-\delta)) d v d u\right| \\
\leq & |p(t) x(t-\tau)-p(s) x(s-\tau)| \\
& +\left|\int_{s}^{\infty} \frac{1}{r(u)} \int_{u}^{\infty} f(s, x(v-\delta)) d v d u\right| \\
\leq & 2 e^{-K_{2}(t-\tau)}+\left(p(t) e^{K_{1} \tau}-1\right) e^{-K_{1} t} .
\end{aligned}
$$

Since $e^{-K_{1} t} \rightarrow 0$ and $e^{-K_{2}(t-\tau)} \rightarrow 0$ as $t \rightarrow \infty$, we conclude from the above inequalities that, for any given $\varepsilon>0$, there exists $t_{3} \geq t_{2}$ such that for all $x \in \Omega$, and $s, t \geq t_{3}$,

$$
|(F x)(t)-(F x)(s)|<\varepsilon .
$$

This means that $F(\Omega)$ is relatively compact in the topology of the Fréchet space $C\left[t_{0}, \infty\right)$.

Similarly to Theorem 2 in [4] and using Schauder's fixed point theorem we can conclude that there exists an $x \in \Omega$ such that $x=F x$. That is, $x(t)$ is a positive solution of (36) and thus also of (1). Since $\lim _{t \rightarrow \infty} x(t)=0$, by Lemma 2, it follows that $\lim _{t \rightarrow \infty} y(t)=0$ and $\lim _{t \rightarrow \infty} y^{\prime}(t)=0$. The proof is complete.

We close this paper by remarking that it would not be difficult to extend all the results in this paper to an equation whose nonlinear term has the form

$$
f\left(t, x\left(t-\delta_{1}\right), \ldots, x\left(t-\delta_{m}\right)\right)
$$


Acknowledgements. I am grateful to Professor Sui Sun Cheng for his assistance.

\section{References}

[1] I. Gyori and G. Ladas, Oscillation Theory of Delay Differential Equations with Applications, Clarendon Press, Oxford, 1991.

[2] H. J. Li and W. L. Liu, Oscillations of second order neutral differential equations, Math. Comput. Modelling 22 (1995), 45-53.

[3] -, 一, Oscillation criteria for second order neutral differential equations, preprint.

[4] W. D. Lu, Existence and asymptotic behavior of nonoscillatory solutions of second order nonlinear neutral equations, Acta Math. Sinica 36 (1993), 476-484.

[5] J. Ruan, Types and criteria of nonoscillatory solutions of second order linear neutral differential equations, Chinese Ann. Math. Ser. A 8 (1987), 114-124.

Department of Mathematics

Zhangye Teachers' College

Zhangye, Gansu 734000

P.R. China 